Sessions d'étude - Société canadienne d'histoire de l'Église catholique

\title{
Les positions religieuses de Moses Hart
}

\section{Denis Vaugeois}

Volume 33, 1966

URI : https://id.erudit.org/iderudit/1007320ar

DOI : https://doi.org/10.7202/1007320ar

Aller au sommaire du numéro

Éditeur(s)

Les Éditions Historia Ecclesiæ Catholicæ Canadensis Inc.

ISSN

0318-6172 (imprimé)

1927-7067 (numérique)

Découvrir la revue

Citer cet article

Vaugeois, D. (1966). Les positions religieuses de Moses Hart. Sessions d'étude -

Société canadienne d'histoire de l'Église catholique, 33, 41-46.

https://doi.org/10.7202/1007320ar

Tous droits réservés @ Les Éditions Historia Ecclesiæ Catholicæ Canadensis Inc., 1967
Ce document est protégé par la loi sur le droit d'auteur. L'utilisation des services d’Érudit (y compris la reproduction) est assujettie à sa politique d'utilisation que vous pouvez consulter en ligne.

https://apropos.erudit.org/fr/usagers/politique-dutilisation/ 


\section{Les positions religieuses de Moses Hart}

Comme Moses Hart n'appartient pas - du moins pas encore à la grande histoire, il convient de le présenter brièvement.

Moses ou Moïse Hart est le fils aîné d'Aaron Hart et de Dorothée Judah. On sait qu'Aaron est généralement considéré comme le premier Juif à s'être établi au Canada.

Retenons que le père fut un Juif de stricte observance et qu'il s'occupa durant toute sa vie - et ce malgré ses multiples affaires d'organiser la communauté juive des Trois-Rivières. On sait aussi qu'il s'est fortement préoccupé de l'instruction et de l'éducation religieuse de ses quatre fils : Moses, Ezekiel, Benjamin et Alexandre. Seul le dernier devait épouser une chrétienne. Les trois autres, de même que ses trois filles qui, à notre connaissance, ont prix époux, ont fait des mariages juifs.

Tous furent citoyens en vue et jouèrent un rôle important, en particulier pour la collectivité juive. La loi d'Ezekiel servira d'ailleurs de prétexte à ses adversaires pour l'empêcher de siéger à l'assemblée législative du Bas-Canada. Benjamin jouera un rôle prépondérant à la Sherith Israël de Montréal et contribuera fortement à l'émancipation des Juifs du Bas-Canada. Les époux des trois sœurs Hart seront aussi des Juifs bien en vue.

Tout ceci pour bien montrer que Moïse est d'une famille où la conscience juive a été clairement affirmée.

Né en 1768, Moïse épousait en 1799 sa cousine Sarah Judah après des fréquentations fort mouvementées, agrémentées particulièrement de prises de bec entre futurs gendre et beau-père.

Moïse ne fut jamais heureux en ménage. Il est assez difficile de savoir si son mariage fut une faillite - la seule de son existence d'ailleurs - à cause de son tempérament, ou si, au contraire, cet échec matrimonial fut à l'origine de sa conduite pour le moins extravagante.

Il nous semble plutôt qu'il était peu apte à réussir un bon mariage. Quoi qu'il en soit, son épouse obtint de la Cour une première séparation de corps en 1807, reprit la vie conjugale un peu plus tard et revint de nouveau devant les tribunaux en 1814.

Après la mort de sa femme en 1826, Moïse, sans contracter mariage, vécut avec une veuve, Mary McCarthy. Il la reconnut toutefois comme

La principale source de documentation pour cette brève étude fut le Fonds Hart qui est conservé aux Archives du Séminaire des Trois-Rivières. Il faut aussi souligner les études du Dr Jacob Rader Marcus et de M. Raymond Douville. 
sa légataire universelle, de même qu'il reconnut dans son testament quelques-uns de ses enfants naturels.

Il y aurait beaucoup à dire sur les enfants de Moïse, particulièrement sur ses très nombreux illégitimes. Qu'on retienne cependant qu'ils ont existé, car ils permettent de comprendre bien des choses.

Cette vie amoureuse agitée amena sans doute Moïse à s'interroger sur sa religion et celles des aurtes. Comme on peut supposer que ses incartades soulevaient bien des protestations dans son entourage, il s'en prit violemment aux catholiques qui l'entouraient.

Il ne faut pas s'y tromper, en même temps qu'il dénonçait dans ses écrits le christianisme, il prêtait sans intérêt des sommes respectables aux Ursulines des Trois-Rivières, au point même d'avoir droit à leurs prières comme généreux bienfaiteur. Il finançait aussi de nombreuses paroisses. Moïse Hart ne fut-il pas l'un des plus importants brasseurs d'affaires de la première moitié du $\mathrm{XIX}^{\bullet}$ siècle. Il prêta de l'argent à plusieurs curés et contribua à la construction ou à la restauration de quelques églises dont celles de Saint-Michel d'Yamaska, Saint-Stanislas, Sorel, Saint-Apollinaire, Grondines, Baie de Fèbvre, Saint-Jean Deschaillons.

Sans doute influencé par ses déboires matrimoniaux et ses nombreuses aventures sentimentales d'une part et la lecture des philosophes étrangers d'autre part, Moïse Hart entreprit donc d'étudier et d'attaquer la religion catholique.

Il ne convient pas de s'attarder sur cet aspect: le temps aussi bien que le respect de cet auditoire nous l'interdisent. Mentionnons seulement l'existence de certains textes manuscrits.

Un premier, long de 46 pages, s'intitule : « The ten commandments of the Roman Catholich Chaurch humbly inscribed to the Consideration of the Roman Catholichs of Ireland, Canada and the United States. *

Dans une courte préface, l'auteur se dit fort étonné de voir tant de gens adhérer à la religion catholique. Les trois quarts des Irlandais et des Canadiens la pratiquent. Ils prétendent s'inspirer du Christ, écrit-il, mais ni lui ni ses disciples n'ont jamais précisé un rite ou une observance. Il poursuit dans la même veine et demande humblement la permission de rédiger quelques pages dans lesquelles il va tenter de retracer les origines et les différentes étapes de la foi catholique; à quelles affinités elle peut prétendre; à ses rapports avec le Christ et la Révélation, comme avec la Raison; à son influence sur le monde, la littérature, les arts, les sciences et le commerce.

Toujours dans sa préface. il s'en prend à « ce détestable principe qui veut que toute personne d'une autre religion soit condamnée aux flammes éternelles ». " Je crie avec horreur, écrit-il encore, devant le flot de sang innocent que cette religion a fait couler en Europe....

Et enfin, il en vient aux dix commandements, annoncés dans le titre. Qu'il nous suffise de les énumérer : 
- Au pape tout comme au Christ, tu obéiras.

II - Les évêques ne se souilleront pas au contact d'une femme.

III - De même les prêtres et les diacres ne permettront pas à leur sainte personne les vils rapports d'une femme.

IV - Tu établiras des communautés de Pères et de Frères servants.

$\mathrm{V}$ - Religieuses et prêtres de jupons tu protègeras.

VI - Tu t'adonneras à l'idolâtrie.

VII - Tu confesseras tes péchés au prêtre et tu prieras dans une langue que tu ne comprends pas.

VIII - Tu t'abstiendras de viande pendant les quarante jours du Carême.

IX - Tu assisteras aux messes et vêpres fidèlement les dimanches et jours de fêtes.

X - Tu établiras une Inquisition pour rassasier la cruauté du clergé.

Pour chacun de ces commandements, Moïse prend le temps de s'expliquer, voire de se justifier; il le fait dans un style à la fois emphatique et satirique. Il termine ce pamphlet en affirmant que la religion catholique est constituée des éléments suivants: de cruauté $(2 / 8)$, d'idolâtre $(2 / 8)$, de tyrannie des prêtres $(1 / 8)$, de pénitence $(1 / 8)$ et de superstition $(2 / 8)^{1}$.

Dans un autre texte, il défendra les Juifs contre l'accusation d'avoir voulu la mort du Christ (The Jews vindicated from the assersion cast upon them of having conspired the death of Christ) ou encore il préviendra ses coreligionnaires contre « le précipice de la chrétienté * (An admonition to the Jews to avoid the precipice of Christianity). Moïse Hart dans un texte de plus de 40 pages procède à une étude comparée des 4, évangiles et à une analyse du Pentateuque, pierre angulaire du Christianisme, dont le Talmud fait largement mention note-t-il. Il rappelle que ces 5 livres sont faussement attribués à Moïse

1 Au début de son pamphlet, Moïse avait dressé la liste suivante: idolâtrie \%, cruauté $1 / 8$, pénitence $2 / 8$ et cérémonie $2 / 8$. Il devait par la suite rayer cette première énumération pour la reprendre dans sa conclusion et la modifier.

Les dix commandements (version originale anglaise):

I - Thou shall pay obedience to the pope as to Christ.

II - Thy Bishops shall not defile themselves with a wife.

III - Thy Deacons, and under priests, likewise shall not stain their holy persons, with the vile embraces of wife.

IV - Thou shall establish Monks, and Fryars.

V - Thou shall cloister Nuns, or priests in petticoats.

VI - Thou shall commit idolatry.

VII - Thou shall confess thy sins, to the priests, and shall pray in a language, thou does not understand.

VIII - Thou shall not eat meat, forty days in lent.

IX - Thou shall attend Masses and Vespers and (sic) Saith holidays.

$X$ - Thou shall establish an Inquisition to glut the cruelty of thy Clergy. 
puisqu'ils furent compilés d'après une tradition orale fragile, qui s'était perpétuée chez les Juifs en captivité.

L'histoire de la Vierge Marie le préoccupe particulièrement. Il en parle en termes fort irrespectueux pour conclure que saint Luc et ses collègues, par cette histoire de l'Esprit Saint, ont sans doute voulu cacher l'immoralité de deux femmes: Marie et Elizabeth. Il s'inquiète beaucoup de la réaction de saint Joseph et s'écrie à un moment donné " a strange composition this Holy Ghost ".

Evidemment on ne s'étonnera pas de trouver sous la plume d'un Juif une sévère critique du Nouveau Testament, en même temps qu'une large connaissance de l'Ancien.

A plusieurs reprises, Moïse s'intéressa à l'histoire de la Papauté et entreprit d'en retracer les grandes lignes pour dégager évidemment la faiblesse de certains papes et l'absence de filiation spirituelle, soulignant de longues périodes d'obscurantisme.

Tous ces essais devaient pourtant amener Moïse à proposer non pas le Judaïsme à tous les chrétiens, mais une religion nouvelle à tous les hommes sincères.

La première édition de son traité de religion qui date de 1815 porte le titre General Universal Religion. Par les états de compte qui ont été conservés, nous savons que cette brochure de 60 pages, imprimée par Van Winkle et Wiley à New York, avait coûté $\$ 68.75$ pour 500 exemplaires. Trois ans plus tard, il faisait réimprimer 250 exemplaires et en 1824, il reprenait son traité sous le nouveau titre Modern Religion faisant encore une fois exécuter son travail à New York mais par un nouvel imprimeur, Johnstone et Van Norden.

Dans une note qui termine la seconde édition, l'auteur nous renseigne sur lui-même, sur ses intentions et sur les caractéristiques principales de son "Universal Religion ". Il propose sa religion nouvelle aux Juifs comme aux Déistes, l'établissant sur des principes naturels. Son éducation juive l'a amené à l'étude de l'Ancien et du Nouveau Testaments. Les cinq livres attribués à Moïse ont été compilés par Esdras (Ezra) d'après une tradition incertaine. Sur les auteurs de deux Testaments, on ne sait rien. Particulièrement on ignore tout des évangélistes: leurs sources, leurs origines, leur langue, etc.

Dans son livre, annonce-t-il lui-même, il offre une religion qui autorise une journée de repos par semaine, et trois fêtes principales; les prières sont brèves et absentes de répétitions, les cérémonies sont morales, pratiques et musicales. De façon générale, il ne flatte pas les hommes en exploitant leur naïveté par des récits de miracles et ne tente pas de diminuer le Créateur par des propos familiers.

A l'âge de 15 ans pour les hommes et de 14 pour les femmes, on peut adhérer à sa religion en prêtant un serment d'entrée. Après avoir indiqué les trois devoirs supérieurs de l'homme vis-à-vis Dieu et son 
prochain, il énumère les 25 devoirs secondaires obligatoires. Puis, c'est la cérémonie du mariage avec les engagements appropriés ${ }^{2}$ - à noter que si un ou des enfants sont déjà nés hors mariage, ils assisteront à la cérémonie placés entre les parents. Vient ensuite le mécanisme de divorce que suppose une séparation préalable de six mois et une cause portée devant jury. Si tout va bien, les conjoints pourront se remarier après dix-huit mois. Sinon, il y a toute une série de délais prévue.

Survient une série de prières: pour la mère qui doit accoucher, pour celle qui vient d'être libérée, pour la cérémonie « d'appellation ", la prière du matin et du soir, pour le jour de repos, les jours de fêtes, les malades, les repas, les voyages en mer, les sépultures, la paix, etc.

Dans une sérieuse étude publiée en $1947^{3}$, le Dr. Marcus s'interrogeait sur les influences reçues par Hart et sur celle que lui-même a eue. Selon ce savant spécialiste, Hart était bien informé des écrits des philosophes français et américains; l'influence de Thomas Paine est évidente. Moïse possédait d'ailleurs quelques-uns de ces écrits que ses descendants ont conservés.

Le Dr. Marcus se demande aussi où se situe Hart parmi les Réformateurs du judaïsme. S'il semble avoir peu d'affinité entre ceux de Charleston et Hart, ou avec le père du mouvement réformiste juif, Israël Jacobson ${ }^{4}$, il n'en va pas de même pour David Friedländer qui, comme Moïse Hart, discutait la valeur du Pentateuque et croyait que le Juif avait été à l'origine déiste.

Il ressort que Moïse Hart avait pu, bien que Bas-Canadien, recevoir l'influence du déisme et du libérationisme de son époque. Est-ce à l'occasion de ses nombreux voyages? C'est bien possible!

Chose certaine, il avait passablement lu. Et c'est peut-être Malthus qui lui a suggéré l'idée de considérer la mort comme nécessaire afin de faire de la place pour ceux qui suivent.

Quelle influence eut-il? Assez minime, semble-t-il. Bien qu'il ne soit pas du tout impossible qu'il ait influencé certains réformateurs juifs du début du XIX` siècle. De son côté, il a pris tous les moyens pour faire connaître ses écrits. Il envoie sa brochure un peu partout; il utilise ses contacts d'affaire, ses parents et amis. Plusieurs lui demanderont de

2 Moses Hart, Universal Religion, page 17: « When a boy arrives at the age of fifteen, and a girl at fourteen, they may contract marriage. In order to lessen the jealousy of married men, the bride is to take an oath of fidelity. This is not exacted from the bridegroom, as some countries admit of polygamy. Indeed, human nature appears inclined to polygamy; however, it rarely happens when the wife is true to, and fulfils her duties to her husband, that he is not actuated by a faithful and tender demeanor toward the mother of his children. $D$

3 Jacob Rader Marcus, The Modern Religion of Moses Hart, Hebrew Union College Annual, vol. XX, Cincinnati, 1947.

4 Ibid, p. 599-600. 
cesser ses envois, sous prétexte que le marché possible de la région concernée est maintenant saturé ${ }^{5}$.

A maintes reprises (1815-1825), il se rendit lui-même aux EtatsUnis pour discuter de sa religion, il entretint une correspondance avec des déistes américains, dont William Carver, un M. Foster et un Dr. Cook. On a conservé le texte d'une allocution qu'il aurait prononcée en novembre 1821 à Albany où il parle de sa religion nouvelle, se justifie comme auteur d'une telle initiative, proteste contre l'intolérance reliGieüise êt iéclant la liberié ioiale.

Après 1825-1826, il semble avoir retrouvé un certain calme. Peut-être Mary McCarthy y est-elle pour quelque chose! Il voit maintenant à ses nombreuses affaires et s'intéresse plus aux questions politiques qu'aux questions religieuses.

Il y va de ses dons à la Sherith Israël de New-York et de Montréal. A sa mort, il reçoit une sépulture juive. Le rabbin montréalais, Abraham de Lola, est l'officiant. Son frère Benjamin, alors trésorier de la synagogue montréalaise, suggère à Mme McCarthy de payer pour le repos de l'âme de Moïse un escoba perpétuel; il en coûtera $\$ 100.00$.

Malgré ses tendances religieuses et ses velléités de réforme, Moïse Hart était donc demeuré fidèle à la religion de ses Pères. S’il n'avait pu léguer aux siens une religion nouvelle, il leur laissait une immense fortune dont les seigneuries de Courval, de Sainte-Marguerite, des Ecureuils, de Gaspé, de Bélair, de Saint-Aimé, les fiefs Godefroy et VieuxPont, le marquisat du Sablé, etc.

Telles furent - très brièvement esquissées - les idées religieuses de l'être le plus extraordinaire et surtout le plus extravagant que nous ayons rencontré.

\author{
Denis Vaugeois \\ Chef de la Division de l'Histoire, \\ Ministère de l'Education, \\ Québec, P.Q.
}

5 Samuel Judah à Moses Hart, New Brunswick, ler septembre 1818, Fonds Hart, A-G-4: "I have only sold three of the books you sent me and I do not perceive any chance of my disposing of the others. If you have an agent in New-York, perhaps he would do better with them than I can. When you was here last, you told me you had written a work on Catholicism... 》. Robert Christie à Moses Hart, Quebec, 13th April 1816, Fonds Hart A-G-3 : a I have shown your Universal Religion to one or two of my private friends who much approve of it with myself as a good moral system.... ». En post-scriptum, il ajoute : "I am about establishing a Printing Press in Quebec, if it can be of any service to you... etc. o Edward Kercheval à Moses Hart, Yellow Spring, October 10th, 1826, Fonds Hart, A-G-2 : «I do a little regret that I paid 25 cents for a letter directed to the Yellow Spring Community accompanied with a small pamphlet intitled Modern Religion but ought to have been titled Modern Deism or a blow at the very foundation of Christianity - which many have aimed at before you-but fortunately for the Christian - the combined forces both of earth and hell can never shake the foundation on which the Christian stand - (...). 